\title{
Involvement in Fusion Activity of an Epitope in the S2 Subunit of Murine Coronavirus Spike Protein
}

\author{
${ }^{1}$ FUMIHIRO TAGUCHI AND ${ }^{1,2}$ YOHKO K. SHIMAZAKI \\ ${ }^{1}$ National Institute of Neuroscience, NCNP, 4-1-1 Ogawahigashi, Kodaira, Tokyo 187-8502; \\ ${ }^{2}$ National Veterinary Assay Laboratory, 1-15-1 Tokura, Kokubunji, Tokyo 185-8511 Japan
}

\section{INTRODUCTION}

The spike projecting from the virion of mouse hepatitis virus (MHV) is composed of a spike (S) glycoprotein with 180 to $200 \mathrm{kDa}$. The $\mathrm{S}$ protein is cleaved into N-terminal S1 and C-terminal S2 subunits. The S1 forms the globular part of the spike and the S2 its stalk portion. An important biological function of the $\mathrm{S}$ protein is binding to the virus receptor, which is mediated by a domain located in the $\mathrm{N}$ terminal 330 residues of the $\mathrm{S} 1$ (S1N330) (Kubo et al., 1994). Virus-cell fusion is also mediated by the S protein (Collins et al., 1982). Various regions in the S2 are involved in fusion activity. A candidate fusion peptide was reported to reside in the S2 (Luo and Weiss, 1998).

A monoclonal antibody (MAb) 5B19.2, which recognizses an epitope A (S2A) composed of nine hydrophobic amino acids in the S2 (Luytjes et al., 1989), has virus neutralizing (VN) and fusion inhibition (FI) activities (Collins et al., 1982). Antibodies to the S2A play a part for protection in mice (Koolen et al., 1990). These findings suggest that the S2A plays an important role in either virus-receptor interactions and/or viral entry into cells. In this study, we investigate the involvement of the S2A in the receptor-binding and fusion activities by using $\mathrm{S} 2 \mathrm{~A}$ antiserum with $\mathrm{VN}$ and FI activities as well as S proteins with mutated S2A.

The Nidoviruses (Coronaviruses and Arteriviruses).

Edited by Ehud Lavi et al., Kluwer Academic/Plenum Publishers, 2001. 


\section{MATERIALS AND METHODS}

\subsection{Cells, viruses and antibodies}

DBT and RK13 cells as well as two MHV JHMV variants, cl-2 and sp-4 (Taguchi and Fleming, 1989), were used. The recombinant vaccinia virus vTF7.3 encoding the T7 RNA polymerase was used to express the JHMV S protein or MHV receptor protein. S2A antiserum ( $\alpha-\mathrm{S} 2 \mathrm{~A})$ was made using rabbits by a synthetic peptide corresponding to amino acids 896 to 911 of JHMV cl-2 S protein (Taguchi et al., 1992). MAb 5B19.2 was provided by M. J. Buchmeier (Collins et al., 1982). S1-specific MAbs 3, 6, 7, 13, and 93 recognize conformational epitopes located in the S1N330 (Kubo et al., 1993).

\subsection{Construction of $\mathrm{S}$ genes with mutations in the S2A and their expression}

Mutations in the S2A shown in Fig. 1 were created by polymerase chain reaction principally as described previously (Taguchi, 1993) using a pair of primers containing the desired mutations. The S proteins with mutated S2A were expressed in DBT cells as described previously in vaccinia virus-T7 system (Feurst et al., 1986; Suzuki and Taguchi, 1996).

\section{RESULTS}

\subsection{VN and FI activities of S2A antiserum}

MAb 5B19.2 recognizes the S2A and displays VN and FI activities, suggesting that the $\mathrm{S} 2 \mathrm{~A}$ is involved in receptor-binding and/or fusion activities. To confirm that anti-S2A serum has VN and FI activities and also to test above possibility, we have raised $\alpha$-S2A by immunising rabbits with a synthetic peptide. We compared the VN and FI activities of the $\alpha$-S2A with those of 5B19.2 and anti-S1 MAbs. Both $\alpha$-S2A and 5B19.2 showed very low VN titers. Their specific VN values (VN titer/ELISA titer) were $10^{3}$ to $10^{4}$-fold lower than the VN values of anti-S1 MAbs. In contrast, their specific FI values (FI titer/ELISA titer) were the same or slightly higher than those of anti-S1 MAbs. These suggest the $\alpha-\mathrm{S} 2 \mathrm{~A}$ and 5B19.2 neutralize JHMV by a different mechanism than anti-S1 MAbs. 


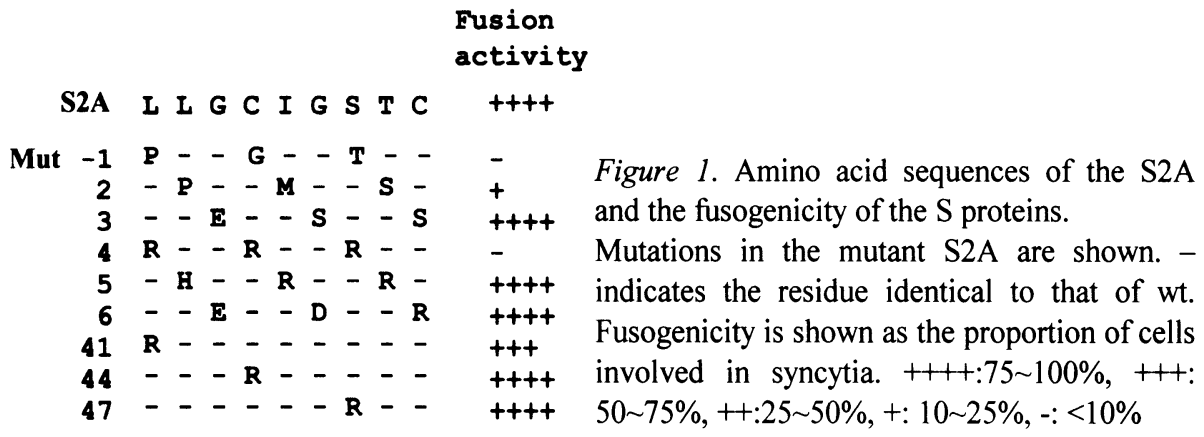

\subsection{Effects of S2A antiserum on receptor binding by JHMV}

We examined whether the neutralization by $\alpha-\mathrm{S} 2 \mathrm{~A}$ is due to the inhibition of the binding of virus to the receptor, CEACAM1 ${ }^{\mathrm{a}}-2 \mathrm{~s}$ (MHVR1). We treated 1-5 $\times 10^{5}$ PFU of JHMV with $20 \mathrm{VN}$ units of various antibodies at room temperature for $1 \mathrm{~h}$. Twenty VN units neutralized more than $95 \%$ of $5 \times 10^{5}$ PFU JHMV. The treated viruses were examined by a viral overlay protein blot assay (VOPBA) using MAb 7 to determine whether they bound to MHVR1; neither the $\alpha$-S2A nor the anti-S1 MAbs used in this study prevents the binding of MAb 7 to $S$ protein. Bands of 40 to $50 \mathrm{kDa}$ corresponding to the MHVR1 were visible when viruses were treated with either MAb 13, $\alpha$-S2A, or normal rabbit serum, indicating that these antibodies fail to block the binding of virus to the receptor (Fig. 2). In contrast, no or only a faint band was produced after treatment with MAbs 3, 6 , and 93, indicating these MAbs effectively block the receptor binding of the virus. Even $200 \mathrm{VN}$ units of MAb 13 failed to block the binding (Fig. 2).

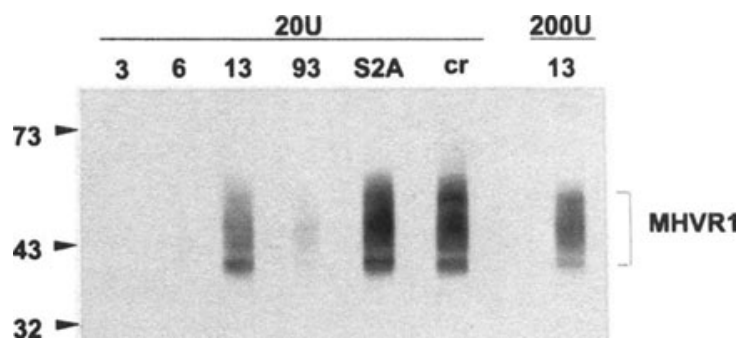

Figure 2. Inhibition of the receptor-binding of JHMV by various anti-MHV S antibodies as examined by VOPBA. Viruses were treated with $20 \mathrm{VN}$ units (U) or $200 \mathrm{U}$ of $\alpha-\mathrm{S} 2 \mathrm{~A}$, MAbs $3,6,13,93$ or normal rabbit serum (cr) and allowed to bind to MHVR1 prepared on membrane paper. 
These results suggest the failure of $\alpha$-S2A to block the binding of virus to the receptor is not due to low antiviral titer but rather because the S2A is not involved in receptor binding.

\subsection{Fusion activity of the $S$ proteins with mutations in the S2A}

To evaluate the role of the S2A in the process of virus-cell fusion, we have prepared a series of mutant $\mathrm{S}$ proteins with amino acid substitutions in the S2A (Fig. 1). In mut-1 to mut-3, three hydrophobic residues were replaced by different hydrophobic ones, while they were changed to hydrophilic ones in mut-4 to mut-47. These mutated S proteins were expressed in DBT cells by vaccinia virus-T7 system. Syncytia formation was monitored by staining with Giemsa solution (Kubo et al, 1993). There was no difference in transfection efficiency of the plasmids containing various $\mathrm{S}$ genes as examined using a vector, pG1NT7 $\beta$-gal, which encodes $\beta$ galactosidase downstream of the T7 promoter. It was shown that mut-1 and mut-4 almost completely lost the fusion activity and the fusogenicity of mut2 and mut-41 was strongly and moderately reduced, whereas the other mutants displayed the fusion activity to the similar extent as wt $\mathrm{S}$ protein (Fig. 1 and 3). These results suggest that $\mathrm{Leu}_{1}, \mathrm{Cys}_{4}$ and $\mathrm{Ser}_{7}$ influence the fusogenicity of the $\mathrm{S}$ protein, with $\mathrm{Leu}_{1}$ being the most important. Other residues were also important for fusion activity, as shown by the reduced fusogenicity of mut-2. Furthermore, these results suggest that the hydrophobicity of the S2A is not critical for its fusion activity.

We have examined the mutant $\mathrm{S}$ proteins expressed in DBT cells by Western blot. Both uncleaved and cleaved forms of $\mathrm{S}$ proteins were detected, though cleavability differed among the mutants. Differences in cleavability did not correlate with differences in fusogenicity (data not shown), consistent with our previous finding (Taguchi, 1993). We further found that wt as well as mutant $\mathrm{S}$ proteins were similarly expressed on the cell membrane (data not shown).

These findings rule out the possibility that the failure of mut- 1 and mut- 4 to induce fusion and the reduced fusion activity of mut- 2 and mut- 41 are due to impaired transport onto the cell membrane. Taken together, our analysis of mutations in the S2A support the idea that the S2A plays a critical role in fusion formation, despite lacking the features of an ordinary fusion peptide. 

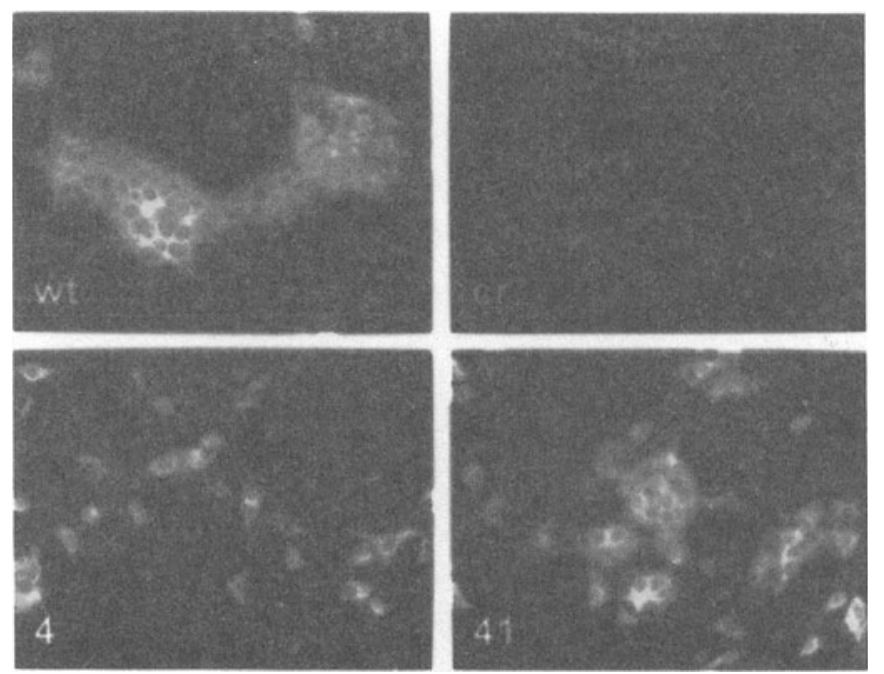

Figure 3. Syncytia formation by the S protein. DBT cells were infected with vTF7.3 and transfected with the $S$ genes of wt, mut- 4 and mut- 41 . The $S$ proteins were detected by the S1 specific MAb at $6 \mathrm{~h}$ posttransfection. $\mathrm{Cr}$ indicates $\mathrm{DBT}$ cells transfected with vector alone.

\section{DISCUSSION}

The present study indicates that the S2A could be involved in fusion activity. In the N terminus of MHV S2, there is no hydrophobic amino acid cluster (Schmidt et al., 1987), which is called fusion peptide generally found in fusogenic enveloped RNA viruses (White, 1990). It was reported recently, however, that a region, PEP1, in heptad repeat 1 of MHV-A59 could work as an internal fusion peptide (Luo and Weiss, 1998). The fusion peptide has several common features, which are conserved in PEP1 but not in S2A. Completion of fusion events for MHV may require several distinct steps of interaction between the viral envelope protein and the cell membrane, with some functions performed by the PEP1 and others by the S2A. Further studies should be undertaken to determine the molecular mechanism by which the S2A participates in fusion events, as well as any specific cellular molecules that may cooperate with the S2A prior to or during fusion events.

\section{CONCLUSION}

MAb 5B19.2 having VN and FI activities binds the S2A consisting of nine hydrophobic amino acids in the S2 of MHV S protein, suggesting the involvement of the S2A in receptor-binding and/or virus-cell fusion. While the binding of virus to the receptor was blocked by the anti-S1 MAbs that 
recognize the receptor binding domain, it was not blocked by the S2A antiserum, indicating that the $\mathrm{S} 2 \mathrm{~A}$ is not involved in receptor binding, nor it influences the receptor binding of the S1N330. S proteins with mutations in a few residues in the S2A were not fusogenic, even when they were replaced by hydrophobic ones, while most $\mathrm{S}$ proteins containing mutations in other residues retained the fusion activity. These results suggest that the S2A is important for the fusion activity of the MHV S protein during viral entry into cells.

\section{REFERENCES}

Collins, A. R.,. Knobler, R. L., Powell, H., and M. Buchmeier, M. J. (1982) Monoclonal antibodies to murine hepatitis virus-4 (strain JHM) define the viral glycoprotein responsible for attachment and cell fusion. Virology 119, 358-371.

Fuerst, T. R., Niles, E. G., Studier, F. W., and Moss, B. (1986) Eukaryotic transient expression system based on recombinant vaccinia virus that synthesizes T7 RNA polymerase. Proc. Natl. Acad. Sci. U.S.A. 83, 8122-8126.

Koolen, J. J. M., Borst, M. A., Horzinek, J. M C, and Spaan W. J. M. (1990) Immunogenic peptide comprising a mouse hepatitis virus A59 B-cell epitope and an influenza virus Tcell epitope protects against lethal infection. J. Virol. 64, 6270-6273.

Kubo, H., Takase, S. Y., and Taguchi, F. (1993) Neutralization and fusion inhibition activities of monoclonal antibodies specific for the $\mathrm{S} 1$ subunit of the spike protein of neurovirulent murine coronavirus JHMV cl-2 variant. J. Gen. Virol. 74, 1421-1425.

Kubo, H., Yamada, Y. K., and Taguchi, F. (1994) Localization of neutralizing epitopes and the receptor-binding site within the amino-terminal 330 amino acids of the murine coronavirus spike protein. J. Virol. 68, 5403-5410.

Luo, Z., and Weiss, S. R. (1998) Roles in cell-cell fusion of two conserved hydrophobic regions in the murine coronavirus spike protein. Virology 244, 483-494.

Luytjes, W., Geerts, D., Posthumus, W., Meloen, R., and Spaan, W. J. M. (1989) Amino acid sequence of a conserved neutralizing epitope of murine coronaviruses. J. Virol. 63, 14081412.

Schmidt, I., Skinner, M., and Siddell, S. (1987) Nucleotide sequence of the gene encoding the surface projection glycoprotein of coronavirus MHV-JHM. J. Gen. Virol. 68, 47-56.

Suzuki, H., and Taguchi, F. (1996) Analysis of the receptor binding site of murine coronavirus spike glycoprotein. J. Virol. 70, 2632-2636.

Taguchi, F. (1993) Fusion formation by uncleaved spike protein of murine coronavirus JHMV variant cl-2. J. Virol. 67, 1195-1202.

Taguchi, F., and Fleming, J. O. (1989) Comparison of six different murine coronavirus JHM variants by monoclonal antibodies against the E2 glycoprotein. Virology 169, 233-235.

Taguchi, F., Ikeda, T., and Shida, H. (1992) Molecular cloning and expression of a spike protein of neurovirulent murine coronavirus JHMV variant cl-2. J. Gen. Virol. 73, 10651072.

White, J. M. (1990) Viral and cellular membrane fusion proteins. Annu. Rev. Physiol. 52, 675-697. 